\title{
PARASITISMO POR CESTOIDES DA ORDEM Trypanorhyncha NA MUSCULATURA DE Plagioscion squamosissimus - PESCADA BRANCA (PERCIFORME: SCIAENIDAE), COMERCIALIZADOS EM MACAPÁ, AP
}

\author{
Antônio Carlos SouZa Silva JúNIOR ${ }^{1}$ \\ 1. Mestrando do programa de pós-graduação em Ciência Animal (Ecologia Aquática e Aquicultura) da Universidade Federal do Pará.
}

RESUMO

O objetivo da pesquisa foi determinar as espécies de cestoides da ordem Trypanorhyncha que parasitam o Plagioscion squamosissimus Heckel, 1840, comercializados nas feiras em Macapá, Amapá. No período entre março de 2008 e dezembro de 2008, necropsiaram-se oitenta espécimes de P. squamosissimus, sendo que sessenta e três peixes $(78,8 \%)$ estavam parasitados por plerocerco da ordem Trypanorhyncha. Registrou-se um poliparasitismo na musculatura do peixe por quatro espécies: Pterobothrium heteracanthum, Callitetrarhynchus gracilis, Poecilancistrium caryophyllum, e Pterobothrium crassicolle. Os blatocistos de Trypanorhyncha na musculatura não apresentam risco de infecção para humanos, mas afetam a estética do pescado, pelo aspecto repugnante, podendo ser rejeitado pelo consumidor e, muitas vezes, tendo a comercialização impedida pela fiscalização sanitária. Este foi o primeiro registro de parasitos de musculatura em peixes provenientes do Estado do Amapá.

PALAVRAS-CHAVES: Amapá, peixe, Trypanorhyncha.

\section{ABSTRACT}

\section{PARASITISM OF CESTODES FROM Trypanorhyncha ORDER IN THE MUSCULATURE OF Plagioscion squamosissimus (PERCIFORME: SCIAENIDAE), COMMERCIALIZED IN MACAPÁ, AP/BRAZIL}

The objective of this research was to determine the species of cestodes from the Trypanorhyncha order that parasites the Plagioscion squamosissimus Heckel, 1840 (Brazilian common name: Pescada Branca), which are commercialized at fairs from Macapá, Amapá State, Brazil. From March 2008 to December 2008, 80 specimens of $P$. squamosissimus were submitted to necropsy, from which 63 fish (78.8\%) were parasitized by larvae plerocercoids from Trypanorhyncha order. A multiple parasitism of four species, Pterobothrium heteracanthum, Callitetrarhynchus

KEYWORDS: Amapá, fish, Trypanorhyncha.

\section{INTRODUÇÃO}

Ocasionalmente, peixes destinados ao consumo humano podem estar infectados por determinadas gracilis, Poecilancistrium caryophyllum, Pterobothrium crassicolle was recorded in the musculature of the fish. Larvae plerocercoids from Trypanorhyncha in the musculature do not present any risk of infection for humans, but affect the aesthetics of the fish, for its repulsive aspect, and may be rejected by consumers and often have the commercialization prevented by the sanitary fiscalization. This was the first record of parasites of musculature in fish proceeding from the State of Amapá.

espécies de parasitos. Alguns podem pôr em risco a saúde dos consumidores, transmitindo-lhe doenças, e outros que, mesmo não sendo transmissíveis ao homem, adquirem importância sanitária quando cau- 
sam um aspecto repugnante no pescado. O resultado é o desprezo daquele espécime parasitado, como, por exemplos, os cestoides da Ordem Trypanorhyncha (SÃO CLEMENTE et al., 1991).

Essa ordem é caracterizada pela presença de um escólex do tipo botridial e em cada um dos botrídios há um tentáculo eversível. O estádio larval, conhecido como plerocerco ou pós-larva, é encontrado nas cavidades corpóreas de peixes teleósteos, crustáceos ou, raramente, em répteis (DOLLFUS, 1942).

Esses cestoides, quando adultos, têm como hábitat o sistema gastrointestinal de peixes elasmobrânquios. Suas formas larvares são encontradas em várias espécies de peixes teleósteos, elasmobrânquios e uma variedade de invertebrados marinhos (CAMPBELL \& BEVERIDGE, 1994).

OVERSTREET (1977) verificou que a aparência da musculatura parasitada por Trypanorhyncha desencorajava muitas pessoas a ingerirem peixes estuarinos no México. Segundo SÃO CLEMENTE et al. (1995), além do aspecto repugnante que esses parasitos conferem ao peixe, a localização de larvas de Trypanorhyncha na musculatura dos peixes pode produzir toxinas que causam reação alérgica na ingestão por pessoas intolerantes.

PALM (1997) observou que as informações sobre o parasitismo por cestoides Trypanorhyncha em peixes da costa tropical brasileira são restritas a poucos trabalhos, sendo a quase totalidade das investigações desenvolvidas na costa Sudeste e realizadas em apenas uma ou poucas espécies de peixes. O presente trabalho teve como objetivo relatar a presença de cestoides da ordem Trypanorhyncha em peixes comercializados na cidade de Macapá, registrando seus aspectos quantitativos e sua importância comercial.

\section{MATERIAL E MÉTODOS}

A Feira do Pescado se realiza em via pública, no Bairro do Perpétuo Socorro. Sua estrutura pode ser dividida em duas partes. De um lado, encontram-se barracas de madeira cobertas, a maioria construída pelos próprios comerciantes, e possuem uma condição precária para a comercialização do pescado. Do outro, veem-se stands mais elaborados, construídos por uma parceria do Governo do Estado com a Associação de Pescadores local. Nas barracas, a venda de pescado fresco eviscerado é predominante, sendo os próprios pescadores ou seus familiares que o comercializam. Nos stands o pescado é vendido por comerciantes, que adquirem o produto de terceiros, o qual é proveniente de outros estados como Pará e Amazonas.

Com o intuito de identificar parasitos nas musculaturas do pescado comercializado na feira, obtiveram-se oitenta exemplares de pescada branca (P. squamosissimus), eviscerada, com os próprios pescadores locais que comercializam o seu produto para a retirada dos filés.

O material coletado foi identificado, embalado em sacos plásticos e acondicionado em caixas isotérmicas contendo gelo. Em seguida, foi transportado para o Laboratório de Ictiologia do Instituto de Pesquisas Científicas e Tecnológicas do Estado do Amapá - IEPA -, onde se realizou a morfometria do pescado (comprimento total $\mathrm{CT}$ - e comprimento padrão - CP) e logo em seguida se efetuou a retirada dos filés. Esta ocorre ao longo de todo o comprimento do peixe, através de uma incisão que se inicia próximo aos opérculos, indo até a inserção da nadadeira caudal, obtendo-se dois filés, um de cada lado.

Os dois filés obtidos de cada exemplar de peixe foram observados em mesa de inspeção do tipo candling table (constituída de um tampo de vidro fosco e de uma luz branca na parte inferior) que, por transparência, permite a visualização dos cistos dos parasitos entre os tecidos examinados.

Os blastocistos presentes na musculatura foram removidos dos tecidos com auxílio de pinça e tesoura cirúrgica e em seguida mensurados. Em estereomicroscópio, o escólex dos cestoides coletados foi liberado do blastocisto, passando por processamento segundo a metodologia-padrão na ictioparasitologia para parasitos da classe Cestoda (AMATO et al., 1991).

Para a identificação e classificação sistemática dos helmintos da ordem Trypanorhyncha, baseaouse nos trabalhos de DULLFUS (1942), REGO et al. (1974), CAMPBELL \& BEVERIDGE (1994) e PALM (1997). As médias e as frequências foram as medidas utilizadas como parâmetros para relatar a prevalência, abundância, intensidade de infecção.

\section{RESULTADOS}

Dos oitenta peixes examinados, que apresentaram variação do comprimento total de $33,43 \mathrm{~cm}$ a 49,61 
$\mathrm{cm}$, com média de 41,24 $\mathrm{cm}$ para a espécie em estudo, $63(78,75 \%)$ apresentaram parasitismo muscular por pelo menos um blatocisto. Nestes, recuperaram-se 345 blastocistos de quatro espécies diferentes, caracterizando um poliparasitismo na espécie, com intensidade média de infecção de 5,48 larvas/peixe e abundância média de 4,31. Os exemplares examinados foram nega- tivos para infecção por outros helmintos. Na Tabela 1 está apresentada a distribuição dos parasitos em relação ao comprimento total dos peixes analisados, demonstrando que ocorreu parasitismo em todas as classes de comprimento. A maior prevalência foi encontrada na classe $45.01-50.00$.

TABELA 1. Distribuição dos parasitos em $P$. squamosissimus em relação ao comprimento total desses peixes, com intervalo de classe de $5 \mathrm{~cm}$, em comparação com sua prevalência em cada classe de comprimento

\begin{tabular}{cccccc}
\hline Comprimento dos peixes & $\mathrm{N}^{\text {o de peixes }}$ & $\begin{array}{c}\text { Comprimento médio } \\
(\mathrm{CM})\end{array}$ & $\mathrm{N}^{\mathrm{o}}$ de parasitos & $\mathrm{N}^{\mathrm{o}}$ de peixes parasitados & $\begin{array}{c}\text { Prevalência } \\
(\%)\end{array}$ \\
\hline $30.00-35.00$ & 17 & 33,76 & 46 & 12 & 70,59 \\
$35.01-40.00$ & 18 & 36,57 & 76 & 14 & 77,78 \\
$40.01-45.00$ & 20 & 42,95 & 98 & 16 & 80,0 \\
$45.01-50.00$ & 25 & 48,32 & 125 & 21 & 84,0 \\
Total & 80 & 41,24 & 345 & 63 & 78,75 \\
\hline
\end{tabular}

O poliparasitismo registrado ocorreu em quatro espécies de cestoides da ordem Trypanorhyncha. A espécie Callitetrarhynchus gracilis (Figura 1) foi a que apresentou maior prevalência $(38,8 \%)$, com intensidade média de infecção de 6,23. Seus blastocistos se apresentavam pequenos $(0,5-1,5 \mathrm{~cm})$, achatados, saculiformes e com membranas delicadas, às vezes, com aspecto claviforme, em vírgula ou com "prolongamento caudal". Os escólex eram filiformes, longos e delicados, com comprimento variando de $0,6-2,8 \mathrm{~cm}$.

A espécie Pterobothrium heteracanthum (Figura 1), que parasitou 23 peixes, apresentou pre- valência de $28,8 \%$ e intensidade média de infecção de 5,35. Os blatocistos apresentaram forma pequena $(0,3-0,6 \mathrm{~cm})$, formato oval, fusiforme ou em vírgula, achatados e com membranas muito delgadas, de tonalidade variando do bege ao vermelho, com uma das faces apresentando uma área transparente. Além disso, geralmente possuem um longo "prolongamento caudal" translúcido. O frágil escólex liberado do blastocisto mostrava-se longo $(2,6-5,7 \mathrm{~cm})$ e estreito, com quatro botrídios bem separados e apêndice bem desenvolvido.

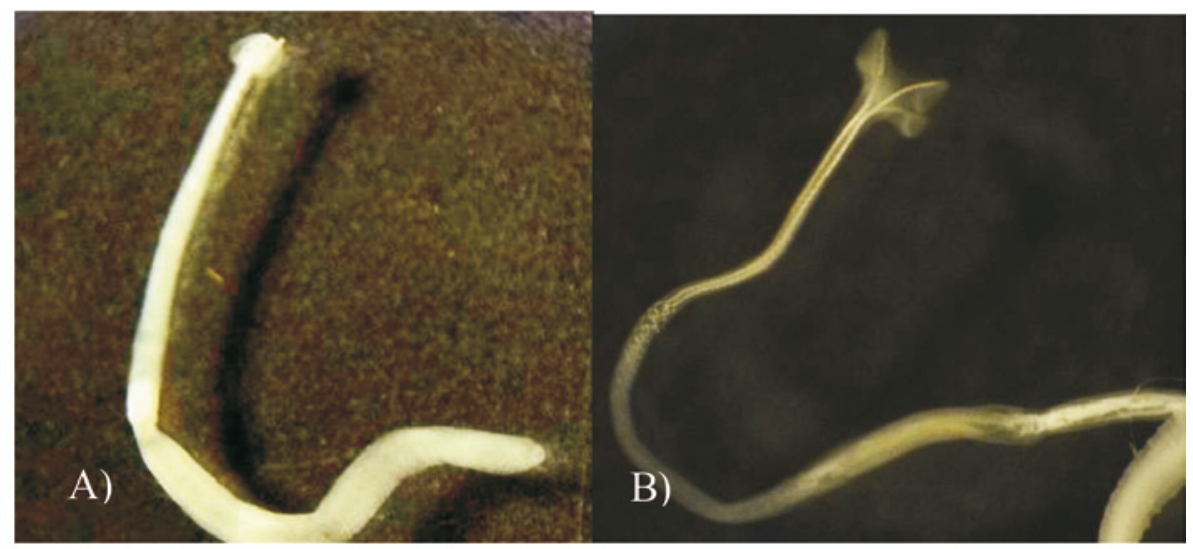

FIGURA 1. Larvas de Callitetrarhynchus gracilis (A) e Pterobothrium heteracanthum (B). 
Também foram encontradas as espécies Pterobothrium crassicolle (7,5\%) e Poecilancistrium caryophyllum (3,75\%) (Figura), que apresentaram intensidade média de infecção de 2,67 e 4,33, respectivamente (Tabela 2 ). O cestoide $P$. crassicolle apresentou blastocistos do parasita muito pequenos $(0,2-0,3 \mathrm{~cm})$ e frágeis, sendo o escólex um pouco mais longo (4-6mm) e robusto, possuindo apêndice curto e quatro botrídios distintos, salientes e arredondados. Para $P$. caryophyllum, os blatocistos eram pequenos $(0,4-0,7 \mathrm{~mm})$, esféricos, brancos, com paredes espessas e longo "prolongamento caudal” $(8-10 \mathrm{~cm})$. Já o escólex era robusto e longo $(0,9-2,7 \mathrm{~cm})$, com apêndice bem desenvolvido e de aspecto sanfonado, com dois volumosos botrídios pateliformes, subcirculares, sobressaindo lateralmente o corpo do escólex.

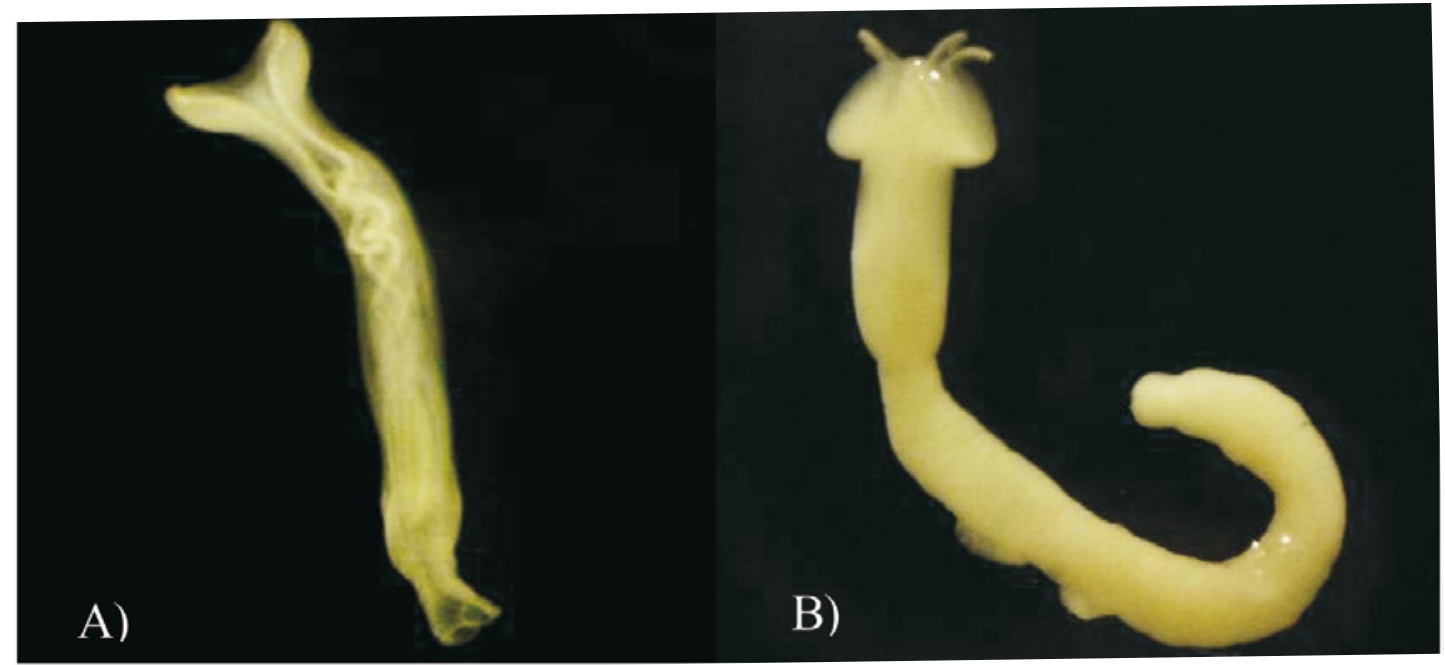

FIGURA 2. Larvas de (A) Pterobothrium crassicolle e (B)Poecilancistrium caryophyllum.

TABELA 2. Espécies de cestoides encontrados em P. squamosissimus e suas respectivas prevalências e intensidade média de infecção

\begin{tabular}{|c|c|c|c|c|}
\hline $\begin{array}{l}\text { Espécie de } \\
\text { cestoides }\end{array}$ & $\begin{array}{l}\mathrm{N}^{\circ} \text { de peixes } \\
\text { infectados }\end{array}$ & $\begin{array}{l}\text { Prevalência } \\
(\%)\end{array}$ & $\begin{array}{l}\mathrm{N}^{\mathrm{o}} \text { de blatocistos } \\
\text { recuperados }\end{array}$ & $\begin{array}{c}\text { Intensidade média de } \\
\text { infecção }\end{array}$ \\
\hline Pterobothrium heteracanthum & 23 & 28,75 & 123 & 5,35 \\
\hline Callitetrarhynchus gracilis & 31 & 38,75 & 193 & 6,23 \\
\hline Poecilancistrium caryophyllum & 3 & 3,75 & 13 & 4,33 \\
\hline Pterobothrium crassicolle & 6 & 7,5 & 16 & 2,67 \\
\hline Total & 63 & 78,75 & 345 & 5,48 \\
\hline
\end{tabular}

Dos 345 blatocistos recuperados, 206 (59,71\%) encontravam-se na musculatura abdominal e 139 blastocistos (40,29\%) foram encontrados na musculatura dorsolateral, sendo considerada uma musculatura com importância comercial.

\section{DISCUSSÃO}

Este é o primeiro relato de parasitismo por larvas de cestoides da Ordem Trypanorhyncha em peixes provenientes do Estado do Amapá. Entretanto, trata-se de resultados que podem ser comparados com os obtidos por outros autores, em diferentes espécies de peixes, de outras localidades.

Relatos de multiparasitismo por Trypanorhyncha foram também referidos em peixes do litoral do Rio de Janeiro por SÃO CLEMENTE (1986a, 1986b) em corvinas (Micropogonias furnieri), por SÃO CLE- 
MENTE et al. (1991) em bagres (Netuma barba), por SÃO CLEMENTE et al. (1997) em anchovas (Pomatomus saltatrix) e por SÃO CLEMENTE et al. (2004) em congro-rosa. Similarmente, no litoral do Estado de Pernambuco, PALM (1997) registrou o fenômeno em quatro das onze espécies de peixes parasitados.

$\mathrm{O}$ alto percentual de parasitismo muscular dos peixes $(78,8 \%)$ indica que essa área corporal pode ser considerada referência para estudos de levantamento epidemiológico de tal parasitose. É o que afirmam diversos autores, como AMATO et al. (1990), SÃO CLEMENTE (1986b), SÃO CLEMENTE et al. (1991), SÃO CLEMENTE et al. (1995), PALM (1997), SÃO CLEMENTE et al. (1997) e SILVA \& SÃO CLEMENTE (2001), relatando que a invasão muscular é rara ou acidental neste grupo de cestoides de peixes teleósteos.

Poucos relatos estimaram índices de parasitismo por cestoides Trypanorhyncha restritos à musculatura parte do corpo de maior valor nutricional e comercial da maioria dos peixes. Altas taxas de infecção por larvas plerocercoides de Trypanorhyncha foram referidas na musculatura abdominal de bonito (Katsuwonus pelamis) (92,7\%) por AMATO et al. (1990).

Entretanto, valores inferiores foram referidos por SANTOS \& ZOGBI (1971), que assinalaram $15 \%$ de infecção em filés de seis espécies de peixes teleósteos. SILVA \& SÃO CLEMENTE (2001) encontraram valores respectivos de $6,72 \%$ e $0,32 \%$ em filés de dourado (Coryphaena hippurus) e ariocó ( $L u$ tjanus synagris). E SÃO CLEMENTE et al. (1995a) observaram que apenas $10 \%$ dos peixes parasitados por Trypanorhyncha apresentaram comprometimento muscular.

Os resultados expressivos encontrados demonstram a importância capital que este estudo assume no âmbito comercial deste peixe, já que, na inspeção do pescado, os metacestoides da ordem Trypanorhyncha, mesmo não possuindo potencial zoonótico, adquirem relevância higiênica. Isso porque o aspecto repugnante ocasionado quando os peixes teleósteos apresentam elevada intensidade desses parasitos na musculatura, no peritônio, no mesentério e no fígado pode acarretar problemas de comercialização, com a fiscalização sanitária, ou serem rejeitados pelo consumidor, já que são visíveis a olho nu.

\section{REFERÊNCIAS}

AMATO, J. R. F.; SÃO ClEMENTE, S. C.; OLIVEIRA, G. A. Tentacularia coryphaenae Bosc, 1801 (Eucestoda: Trypanorhyncha) in the inspection and technology of the skipjack tuna, Katsuwonus pelamis (L.) (Pisces: Scombridae). Atlântica, v. 12, n. 1, p. 73- 77. 1990.

AMATO, J. F. R.; BOEGER, W. A; AMATO, S. B. Protocolos para laboratório: coleta e processamento de parasitos de pescado. Rio de Janeiro: Imprensa Universitária, UFRRJ, 1991.

CAMPBELL, R. A.; BEVERIDGE, I. Order Trypanorhyncha Diesing, 1863. In: KHALIL, L.F.; BRAY, R.A.; JONES, A. (Eds.). Keys to the cestode parasites of vertebrates. Cambridge: $\mathrm{CAB}$ International, 1994. p. 51-148.

DOLLFUS, R. P. Études critiques sur les tetrarhynques du Muséum de Paris. Archives du Muséum National D' Histoire Naturelle, v. 19 , n. 6 , p. 1-466, 1942.

OVERSTREET, R. M. Poecilancistrium caryophyllum and other Trypanorhyncha cestode plerocercoids from the musculature of $C y$ noscion nebulosus and other sciaenid fishes in the Gulf of Mexico. Journal of Parasitology, v. 63, n. 5, p. 780-789. 1977.

PALM, H. Trypanorhynch cestode of commercial fishes from northeast brazilian coastal waters. Memórias do Instituto Oswaldo Cruz, v. 92, n. 1, p. 69-79. 1997.

RÊGO, A. A.; SANTOS, J. C.; SILVA, P. P. Estudo de cestoides de peixes do Brasil. Memórias do Instituto Oswaldo Cruz, v. 72, n. 3/4, p. 187-204. 1974.

SANTOS, C. A. M.; ZOGBI, P. V. La infestacion de peces em Brasil com larvas de Tetrarhynchus fragilis. In: FAO. Fish inspection and quality control. 1971. p. 262-264.

SÃO CLEMENTE, S. C. Plerocercos da Ordem Trypanorhyncha, parasitos de Corvina Micropogonias furnieri (Desmarest) no litoral do Estado do Rio de Janeiro. Atas da Sociedade Biológica, n. 26, p. 29-36. 1986a.

SÃO CLEMENTE, S. C. Prevalência e intensidade média de infecção de plerocercos de Trypanorhyncha parasitando corvina Micropogonias furnieri (Desmarest) no litoral do Estado do Rio de Janeiro. Atas da Sociedade Biológica do Rio de Janeiro, n. 26, p. 37- 40. 1986 b.

SÃO CLEMENTE, S. C.; COELHO, M. R. T.; SERRA-FREIRE, N. M. Cestoides parasitos de Bagre Netuma barba (Lacépede, 1803) pescados no litoral do Rio de Janeiro e comercializados para consumo humano. Arquivo da Universidade Federal Rural do Rio de Janeiro, v. 14, n. 1, p. 27-34. 1991. 
SÃO CLEMENTE, S. C.; KNOFF,M.; PADOVANI, R. E. S.; LIMA, F. C.; GOMES, D. C. Cestoides Trypanorhyncha parasitos de Congro-Rosa, Genypterus Brasiliensis Regan, 1903 comercializados nos municípios de Niterói e Rio de Janeiro, Brasil. Revista Brasileira de Parasitologia Veterinária, v. 13, n. 3, p. 97-102, 2004.

SÃO CLEMENTE, S. C.; LIMA, F. C.; UCHOA, C. M. A. Parasitos de Balistes vetula e sua importância na inspeção do pescado. Revista Brasileira de Ciência Veterinária, v. 2, n. 2, p. 39-41. 1995.
SÃO CLEMENTE, S. C.; SILVA, C. M.; GOTTSCHALK, S. Prevalência e intensidade de infecção de cestoides Trypanorhyncha em Anchovas, Pomatomus saltatrix (L.) do litoral do Rio de Janeiro, Brasil. Parasitologia al Dia, v. 21, p. 54-57, 1997.

SILVA, C. M.; SÃO CLEMENTE, S. C. Nematoides da Família Anisakidae e cestoides da Ordem Trypanorhyncha em filés de dourado (Coryphaena hippurus) e ariocó (Lutjanus synagris) e sua importância na inspeção de pescado. Higiene Alimentar, v. 15, n. 80/81, p. 75-79, 2001.

Protocolado em: 20 dez. 2009 Aceito em: 23 abr. 2010. 